\title{
Management options and parental voice in the treatment of trisomy 13 and 18
}

\author{
Alaina K. Pyle $\mathbb{1}^{1} \cdot$ Alan R. Fleischman ${ }^{2} \cdot$ George Hardart $^{3} \cdot$ Mark R. Mercurio $^{1}$
}

Received: 26 December 2017 / Revised: 11 May 2018 / Accepted: 17 May 2018 / Published online: 6 July 2018

(c) Nature America, Inc., part of Springer Nature 2018

\begin{abstract}
Trisomy 13 and 18 are rare genetic conditions associated with high rates of congenital anomalies, universal profound neurocognitive deficits, and early death, commonly in the first month after birth. Historically, efforts were made to keep these newborns comfortable, but parents were generally not offered medical or surgical interventions. This practice has begun to change in some hospitals, but remains controversial, and a clear consensus between and even within institutions does not appear to exist. This essay presents a summary of current data and an ethical analysis of the question of whether medical and surgical interventions should be offered to parents of newborns with trisomy 13 or 18 . While compelling arguments can be found on both sides, it is here suggested that informed parents should be given a stronger voice in these decisions than has traditionally been the case. In an effort to improve clarity and consistency within single institutions, a process for developing an institutional guideline for management of patients with these, or similar, conditions is presented.
\end{abstract}

\section{Introduction}

Trisomy 13 and 18 (also known as Patau syndrome and Edwards syndrome, respectively) were for many years considered lethal disorders, and medical interventions beyond comfort measures were generally not offered. These rare conditions ( 1 per 10,000 of live births for trisomy 18 and $\sim 0.4$ per 10,000 for trisomy 13) are commonly associated with anomalies of the central nervous system, heart, and gastrointestinal tract, among others, in addition to universal, profound neurocognitive deficits [1]. Since these syndromes were first described in 1960, the field of neonatology has made significant strides in the management of a variety of other neonatal conditions, such as extreme prematurity and congenital heart disease, that were once

$\bowtie$ Alaina K. Pyle

alaina.pyle@yale.edu

1 Department of Pediatrics and Yale New Haven Children's Hospital, Yale University School of Medicine, New Haven, CT, USA

2 Albert Einstein College of Medicine and Children's Hospital at Montefiore, Bronx, NY, USA

3 Columbia University College of Physicians and Surgeons and Morgan Stanley Children's Hospital, New York City, NY, USA considered universally fatal [2,3]. Many infants with disorders once deemed lethal are now often living significantly longer, albeit with a broad range of associated disabilities due to their underlying disease process. This has led to a recognition that the designation of "lethal anomaly" is no longer accurate for many disorders that continue to sometimes be described in that way, including trisomy 13 and 18 [4].

Evidence from recent years shows that some children with trisomy 13 or 18 are able to live for years at home after medical and/or surgical intervention (including cardiac surgery, respiratory support, gastrostomy tubes, etc.), with case reports of individuals living for more than a decade. The mortality rate continues to be high, with studies reporting survival rates of approximately $10-25 \%$ at 1 year, although interpretation of mortality data is complicated by the fact that, at many centers, no attempt is made to prolong the life of these infants $[5,6]$. Thus, there could be a selffulfilling prophecy of nearly universal early mortality in those centers, as has been described earlier for the most extremely preterm newborns [7]. The phenomenon of selffulfilling prophecy is a risk for disorders with high mortality; if one assumes that patients with a certain disorder cannot survive, and thus elects not to offer potentially lifeprolonging interventions, then the low, or zero, survival rate is perpetuated. This, in turn, reinforces the belief that these patients cannot be saved, and attempts to do so are therefore 
not medically or ethically appropriate. Nevertheless, reports have shown that some patients with trisomy 13 or 18 can and do survive the newborn period, and those who survive the first year tend to have improved rates of long-term survival, with around $10-15 \%$ total survival at 5 and 10 years of age [8].

In this essay, we present an overview of arguments for and against offering medical and surgical interventions for patients with trisomy 13 or 18 , and describe a process by which the staff of a medical center can review their approach and develop a clear, coherent, and fair policy. After having completed such a process, the neonatology faculty at Yale New Haven Children's Hospital modified its prior approach, and significantly increased the level of intervention made available. We suggest that patients with trisomy 13 or 18 should be managed as all others in the neonatal intensive care unit, with treatments offered or provided based upon an understanding of the most recent prognostic data, as well as relevant ethical considerations, and an understanding that parental preference, while not determinative in all cases, should be respected.

\section{Outcomes, evolving guidelines, and practice}

As has been rightly stated by many, good ethics begins with good data. While a thorough review of recent survival and morbidity data is beyond the scope of this essay, some important points beyond the survival data noted above deserve mention before a cogent discussion of the ethical issues at play can occur. This is particularly true in a situation such as this, where the prognostic information that many physicians act upon may no longer be valid.

The most recently available mortality data, as described above, demonstrate that at least a significant minority of these patients can survive beyond the newborn period if efforts are made to prolong life. The profound developmental and intellectual disabilities seen in patients with trisomy 13/18 are well documented, but there are reports via parental surveys of some basic milestones such as sitting, self-feeding, and interactive play being met by some children surviving long term $[9,10]$. These are small and largely anecdotal studies, but do provide limited evidence that some survivors are able to make developmental progress. Through these surveys of parents of children with trisomy $13 / 18$, it has been shown that these children are often perceived as being happy and having a good quality of life, as well as being able to interact with their family $[10,11]$.

A significant number of children with trisomy 13 and 18 have been hospitalized and offered procedures across the United States over the past two decades [12]. Between 1997 and 2009 , nearly 2000 patients were admitted (1/3 of which were birth admissions) in each 1 -year time period studied. There were $\sim 2700$ procedures in total performed in the five 1-year observation time periods studied. One question in particular that has become increasingly controversial is whether to offer cardiac surgery to this population, as the benefit is felt to be limited with a high risk of mortality. This has not borne out in recent studies, showing an improved survival benefit after complete repair of congenital cardiac defects [6, 13-15]. Importantly, there is a higher mortality rate than the general population, but carefully selected patients, ideally at $>3$ months of age and $>3 \mathrm{~kg}$, are often able to tolerate the procedure and successfully be discharged home without mechanical ventilation [13].

Professional organizations utilize evolving data to continually adjust their recommendations for optimal management. The International Liaison Committee on Resuscitation (ILCOR) has published guidelines on neonatal resuscitation every 5 years since 2000 , including a section on when it may be appropriate to withhold interventions [16]. Trisomy 13 and 18 were explicitly mentioned in the 2000 and 2005 guidelines, in a list that included anencephaly and infants $<23$ weeks, as examples of conditions with "almost certain early death and when unacceptably high morbidity is likely among the rare survivors" and therefore "resuscitation is not indicated" [16, 17]. Interestingly, for the updated 2010 guideline, trisomy 18 was removed from that category and trisomy 13 remained [18]. The most recent 2015 guideline states simply that "no new data have been published that would justify a change to these guidelines," while reiterating that "under circumstances when outcome remains unclear... the desires of the parents should be supported" [19]. It is here suggested that trisomy 13/18 should fall into this latter category, with the emphasis on shared decision-making based on parental values.

\section{Rationale for withholding medical and surgical interventions}

Withholding medical and surgical interventions from newborns with trisomy 13 or 18 , that is, not offering these interventions to parents, has been a longstanding approach, though in some centers this is clearly evolving. There are several arguments in support of this approach. Perhaps the most common is based upon futility and the obligation not to prolong or increase suffering when there is no potential for long-term benefit to the child.

The term "futile" is commonly used to describe situations wherein efforts under consideration cannot achieve the desired goal. For this term to be used with any coherence, then, one must first determine the desired goal. One can think of many clinical situations wherein a certain intervention may add hours or days to a patient's life, but no more. Whether such an intervention should be seen as futile will be informed by whether the goal is survival for a few 
more days, or survival for additional years. Moreover, there are treatments, such as pain relief, that may not add significantly to the duration of life but have a reasonable chance of increasing the quality of life. Once again, whether these interventions are deemed futile should depend on the determination and articulation of the goals. In the case of trisomy 13 or 18 , the data clearly show that the belief that survival beyond the neonatal period is impossible, or nearly impossible, is mistaken. We do not know how many would survive if maximal efforts were made for each child, but we know without doubt that some of these children already survive. Thus, if impossibility or near impossibility of survival for months or years is the argument for withholding treatment, it does not stand up. Moreover, the use of the term futile is often used inappropriately in settings such as this. A recent statement from multiple critical care societies rightly suggests that the term "potentially inappropriate" rather than futile should be used to describe treatments that have at least some chance of accomplishing the desired goal, which appears to often be the case for infants with trisomy 13 or 18 [20]. However, if the parents' goal is to have an infant that survives without profound cognitive deficits (and to our knowledge there has never been such a survivor), then it seems reasonable to consider medical interventions to be futile, as they cannot achieve that goal.

A central question, then, is: What is the goal? Or, perhaps an even more fundamental question is, who should determine the goal? We suggest that it should be a joint decision by parents and physicians, with the parental values at the center of the discussion. Parents who see value in the ongoing life of a profoundly disabled child and in their interactions with that child should have the option of continued life-sustaining treatments. Conversely, if the parents believe the severe neurodevelopmental impairments would result in suffering and a poor quality of life for their child, their values should be supported, and comfort measures only would be appropriate.

Another argument against offering interventions beyond comfort measures concerns appropriate allocation of limited medical resources. It could be argued that the funds spent on these children would better be used for others with a better prognosis for long-term survival, or survival without profound disability. We will not here argue that careful and fair allocation of resources is not advisable-it surely is. Health care is a limited resource, and though it is not currently being rationed in a systematic way in the United States, there are situations in which cost drives treatment decisions- at the individual, hospital, and national level. The limitations on use of healthcare resources should be discussed openly and transparently, with an eye to optimizing outcomes, improving efficiency, and respecting individual rights. It could well be argued that conditions such as trisomy 13 and 18, with their associated high rate of mortality and poor developmental outcomes, would be an appropriate setting for which to enforce limitations on treatment.

The burdens and potential harm to families, short term and possibly long term, resulting from the care of such a profoundly impaired child, is another argument for withholding interventions. Considerations might include the effect on the marriage and/or on siblings, as well as a significant negative financial impact on the family.

There may be another, more difficult to assess, burden placed on parents that should be considered in this analysis. Among the many emotions that can be experienced by parents in these difficult situations, and potentially among the most painful, is guilt. Specifically, once an offer of medical or surgical intervention is made, parents may feel obligated to "do everything possible," and feel guilty if they choose otherwise. Thus, it could be argued that by making more treatment options for trisomy 13/18 available to parents, physicians may actually be doing them a disservice, whereby parents are emotionally cornered into making a choice they do not truly want. Experience suggests this may indeed sometimes be the case, and thus physicians are well advised to be careful with what they offer to parents and how they offer it. For example, by proactively explaining why many loving parents choose comfort care for infants with these conditions, physicians may be able to assuage some of the parent's guilt associated with this path. The alternative to these thoughtful and open discussions is to deny families a choice that they might want, risking a wellmeaning but paternalistic usurpation of their parental rights.

\section{Rationale for offering intervention}

Over the past century in the United States, attitudes toward those with intellectual and physical disabilities have shifted towards greater acceptance, with increased valuation of their lives. Trisomy 21 serves as a prime example of this change in outlook over time, both societally and within the medical profession. The provision of life-saving medical and surgical intervention for infants with trisomy 21 has evolved since the 1970s, when it was often considered ethically permissible to forego interventions such as duodenal atresia repair due to the known long-term disability $[21,22]$. This, of course, is no longer the case, and surgical intervention for disorders such as duodenal atresia or trachea-esophageal atresia in the setting of trisomy 21 is now considered obligatory. The point is not that disability in trisomy 13 and 18 is similar to that in trisomy 21 , but that our attitudes toward disability have changed. Thus, we might consider the profound disabilities in trisomy 13 and 18 in a new light. Our values as a profession have changed. And, perhaps even more importantly, there has been an increasing recognition that parental values should be 
respected as a primary voice in the decision-making process for children. Moreover, the value of a life with profound disability may be perceived quite differently by parents, the children themselves, and physicians, and the parental perspective is rightfully being given increasing weight $[23,24]$.

Differences in treatment preferences between physicians and families may at least partially be explained by the major differences in their perspectives and experience. Often, the negative sequelae are seen far more frequently by medical providers-during acute decompensations, admission to the hospital, or surgical procedures-and internalized. Parents, conversely, are able to appreciate the positive moments in the home life of a medically fragile child, as well as their struggles. Therefore, some have argued that parents are best situated to assess the quality of life for their child. This is reflected in surveys completed by parents of children with trisomy 13 and 18, which show that many families believe their child has a good quality of life [9-11].

The right of parents to make medical decisions on behalf of their children, while not absolute, is generally perceived to be far-reaching [23]. This right provides a major justification for providing them with all medically indicated options, as their ability to make decisions can be compromised if limitations are placed on the choices offered by the medical team. Parents are presumed to hold the best interest of their child first and foremost in the decision-making process, and they are best positioned to decide what an acceptable long-term quality of life looks like for their family. However, this right should not be seen as absolute; for example, a parental request or demand for a treatment that offers no clear benefit to the child and may cause harm should not be provided. An excellent review of how to manage potentially inappropriate requests for treatment was recently published and is beyond the scope of this paper [20]. In patients with life-limiting conditions such as trisomy 13 and 18, the line at which it is acceptable to let parental wishes override a physicians' recommendation becomes blurred by the lack of clear knowledge regarding efficacy of specific treatments in this population and difficulties with effectively relaying to parents the potential realities of caring for a severely disabled child. Recent outcome data suggesting that interventions such as cardiac repair may in fact prolong life and improve quality of life should inform our discussions with family, as well as our willingness to offer and perform such procedures [25].

The minimization of suffering is a critical shared goal for both physicians and families. The infant's right to receive medical care that could prevent or treat suffering should play a significant role in the decision-making process, especially with less invasive interventions such as nasal cannula, nasogastric feedings, and medications to treat reflux, apnea, or seizures. These interventions can and should be selected on an individual basis, based on the parents' goals of care and the child's medical needs, without obligating the parents or the care team to be "all in or all out."

It should also be noted that for patients with trisomy 13 and 18, there are interventions, such as medically administered nutrition and hydration, which could be considered both life-sustaining and palliative due to the comfort provided by feeding for many infants. Also, there is a clear but rarely discussed possibility that an infant with trisomy 13/18 is also susceptible to reversible processes in the neonatal period, such as respiratory distress syndrome, transient tachypnea of the newborn, hypoglycemia, and so on, which could resolve with routine interventions. Should we allow these babies to die or suffer through potentially reversible processes, because their underlying condition is life limiting and is associated with significant neurodevelopmental impairment? If a family's goals of care are focused on maximizing time with their child alive, it seems reasonable that medical interventions made available should reflect that desire, especially when managing potentially transient processes.

Finally, there is an issue of fairness or justice, a value long held and widely accepted in medical ethics [26]. The principle of justice suggests that we should treat equals equally and unequals unequally. While there can never be a perfect analogy between diagnoses, it is noteworthy that families of pediatric patients with very poor prognoses, including very poor neurological prognoses, are typically given a choice regarding life-sustaining measures. Consider, for example, a newborn or older child with severe hypoxic-ischemic encephalopathy or traumatic brain injury. It is not immediately clear why the parents of children with trisomy 13 or 18 , with a similarly grim neurological prognosis, should not be accorded the same degree of decisional authority. It is the default position in medicine, and rightly so, that all efforts are made to prolong the patient's life and relieve the patient's suffering. When physicians decide to do otherwise, a justification should be required. Most often, when a treatment is available, feasible, and has the potential to prolong life, the justification for non-treatment in the pediatric setting rests on the family's preference. There is no clear justification why children with trisomy $13 / 18$ should be approached differently.

\section{Ethical analysis}

Often in medicine we answer difficult questions based on how we were taught, or on our past practices. This is not a bad start, but it is only a start, and complete reliance on such an approach impedes medical progress. No one should seek to be the physician who treats medical problems in the manner learned decades ago, without frequent consideration of new developments. New data must be considered, and 
old approaches should be re-examined in that light. It is the same with ethical questions, which require of us occasional reconsideration not just of the relevant ethical principles, but also the most current data. In particular, ethical inquiry requires us to reflect on whether our practice is consistent with our own professed values [27]. Moral progress also requires us to occasionally revisit those values themselves.

The right of a patient or a patient's decision-maker to choose a given treatment is limited by a combination of what is medically available and ethically permissible. We do not advocate a system wherein the physician acts solely as parents dictate. On the contrary, it falls to the pediatrician to serve as a check against parental demands when they are clearly opposed to the child's best interest [20]. Physicians should generally not be obligated to provide treatment that they perceive as unethical, but it is important to honestly consider the basis for that perception, and to consider that, in some situations, the parents' values should be determinative. Given our current understanding of the biases inherent in the determination of the quality and value of life of persons with disabilities, we suggest that a pediatrician's refusal to provide a given treatment based on value judgements in the setting of disability should carry less weight than a refusal based on a factual assessment of patient benefit and burden.

There have been compelling arguments made for and against the ethical permissibility of taking non-patientrelated factors such as financial burden, effect on other children in the family, and so on into account when making treatment decisions. The 2016 AAP policy statement on informed consent acknowledges the approach of considering family's interests in addition to the interests of the patient "as long as the child's basic needs, medical and otherwise, are met." [28] Related to this but distinct is the question of whether it is ethically permissible for physicians to consider non-patient-related factors in these treatment decisions. While this complex debate is beyond the scope of this essay, it seems overly paternalistic for a physician to refuse a treatment for the family's sake, if the family themselves do not share that view.

The increasing deference to parental values remains an area of contention, in part due to concern over cases of perceived inappropriate parental demands for interventions that are considered futile (i.e., cannot achieve the stated goal) as well as those that are considered morally objectionable by the clinical providers. This struggle to find a balance between parental rights and the desire to prevent the use of painful and non-beneficial interventions is addressed by the recent AAP Guidance on Forgoing Life-Sustaining Medical Treatment (LSMT). It states that "It may be ethically supportable to forgo LSMT without family agreement in rare circumstances of extreme burden of treatment with no benefit to the patient beyond postponement of death"
[29]. The tension between parents and providers in these extreme cases has resulted in significant moral distress and highlights the need for a mechanism that allows medical providers to stop LSMT when the family refuses. The recent joint statement from five adult critical care groups strives to meet this need by providing a stepwise pathway to resolving "futility" cases and also instructs physicians not to provide truly futile interventions [20]. This need must continue to be met with great caution within pediatrics, to prevent the pendulum from swinging back towards an overly paternalistic model of care whereby parental authority is lost.

Resource allocation is a frequently utilized argument for withholding interventions for children with trisomy 13 and 18. In the United States, there is no standardized policy for any condition of childhood for which care is explicitly limited by an institution, region, or state based exclusively on the cost of the interventions. Justice requires that equals be treated equally, so it seems unfair to enact a guideline that leads to one group of infants being refused interventions due to financial concerns, unless we include all patients with similar prognoses for survival and morbidity. It should also be noted that the amount of resources expended on these infants over time will be very small in comparison to that spent on the much larger numbers of severely debilitated adults in intensive care near the end of life [30]. Nevertheless, we acknowledge that at some point it may be necessary to limit what is provided to whom. Such decisions, however, should be fair and transparent, and not limited to one small group of individuals without justification.

It is not here argued that medical and surgical interventions in the setting of trisomy 13 or 18 should be seen as ethically obligatory. The threshold for considering a treatment obligatory (i.e., should be provided even over parental objection) ought to be high - the benefits to the child should very clearly outweigh the burdens. Given the severity of short-term and long-term prognoses, intervention in this setting does not meet that threshold and should not be sought or provided over parental objection. However, for a treatment to be seen as ethically impermissible, there should be no chance of benefit to the child, or the burdens of treatment should far outweigh the benefits. Here, too, this threshold is not met. The chance for benefit in at least some cases has been demonstrated. What is often left for these patients is the middle ground, wherein the treatment should be considered ethically permissible. If for a given patient a treatment is permissible, even if inadvisable, then parents should be given the choice. If the physicians judge the treatment to be inadvisable, it seems appropriate for the physician to share his/her recommendation on that question with the parents, and the reasoning behind that recommendation [20]. However, this recommendation should not come with a prohibition against choosing another ethically permissible option. 
Reasonable, thoughtful individuals might look at the ethical arguments for and against, and conclude that medical and surgical interventions beyond comfort measures should not be offered for patients with trisomy 13/18, especially in a resource-poor area. However, based on the considerations presented above, we suggest that the arguments in favor of offering, but not requiring, treatment are ultimately stronger. We conclude that interventions should be offered to families of neonates with trisomy 13 and 18, based on the principle of justice, the right to parental authority, respect for parental values, and a lack of compelling evidence that such interventions cannot achieve parental goals of care.

\section{Developing a management guideline}

To combat the risk for significant inconsistencies in therapies offered within single institutions to families of neonates with confirmed trisomy 13 or 18 , it is here recommended that institutions utilize a stepwise process to develop a clear, transparent, and consistent written management guideline. There is an extensive literature on clinical practice guideline development which is beyond the scope of this manuscript [31]. One approach that has been used successfully in the setting of trisomy 13/18 and is applicable to other ethically challenging scenarios in pediatrics, is outlined below.

The first task in answering a question is to clearly identify and articulate the question at hand. Here, the question is: "What interventions should be offered to families of neonates with confirmed trisomy 13 and 18?" This basic question, the relevant data, and relevant ethical principles/arguments, could be presented in a Grand Rounds or similar presentation, at which appropriate stakeholders are present. This should include, at a minimum, clinicians from General Pediatrics, Cardiology, Genetics, Cardiothoracic Surgery, Pediatric Surgery, Obstetrics, Nursing, Ethics, and Neonatology. In addition, representatives of parent/patient advocacy groups would be a valuable addition to the discussion. After soliciting input from stakeholders, a smaller meeting should be held among the individuals who will have the most direct involvement in medical decision-making in the prenatal and newborn period, so as to form a coherent and feasible plan. In our center, we believed this was best accomplished in a meeting that included all faculty and fellows within the neonatology division. There are many different people involved in the care of neonates with trisomy $13 / 18$ but attempting to include all of them in a decision-making forum would be unwieldy and inefficient, thus the decision to limit participation in the follow-up meeting.

Prior to beginning this meeting, it is recommended that all members of the division agree to abide by the final decision of the group, ultimately to be reached by a majority vote. It is important to acknowledge that this is likely be a difficult prospect for some subset of the group, regardless of the decision made, but is necessary to ensure consistent implementation of the final decision. Given the shared attending responsibility and frequent cross-coverage, it would be highly problematic, confusing, and unfair to parents and newborns to have availability of therapies at a given institution vary from one day to the next. There is a real possibility that, through this process, some members of the group could feel coerced into acting against their conscience, by agreement to abide by the majority opinion. Individual hospitals should have a process by which physicians are able to express their conscientious objections, while ensuring that patient management is unimpeded, and no undue burden is placed on other providers [32].

The first portion of the meeting may best be dedicated to summarizing the current data from the literature, standards of care at this and other institutions, and the important ethical arguments. Availability of treatments for patients with trisomy 13/18 at other centers in the region or beyond will be particularly relevant when considering the option of referral. Everyone present should then be invited to offer their opinion and an explanation of their thought process, and to have an open, frank discussion. Of course, it cannot be mandated, but an openness to opposing points of view is an essential component of a successful process. Due to the inherent power dynamic within most teams, those in a position of leadership, including the chair of the meeting, would be well advised to withhold stating an opinion until the others have had the opportunity to articulate theirs. Alternatively, the meeting could be led by an individual entirely outside the group, such as a mediator, an ethicist, or another medical provider in an area unaffected by the topic at hand. The meeting leader should encourage all present to be involved in the discussion, mediate disagreements if necessary, and keep the group focused on the question being discussed.

After a general discussion, specific treatments should be considered individually. Should gavage feeding be offered? Bag-mask ventilation? Mechanical ventilation? Cardiac surgery? At the conclusion of the meeting, the group should have a clear approach for which specific therapies could be made available to parents of neonates with trisomy 13/18 in their facility. It seems wise to state in the guideline itself that the agreed-upon options should generally be made available, but there may be exceptions based upon the specifics of the case. The guideline should then be shared within the institution, and there should be a willingness to revise if new considerations are brought forth, such as new data or newly available therapies. When the process is completed, information regarding the guideline should also then be available to individuals and families who may be 
affected. The preference for utilizing this guideline via a shared decision-making process with the family in the prenatal time period cannot be overstated. Parental decisions are best made with adequate time to contemplate the available information and options, after speaking with any relevant specialists based on their infants' known or expected medical issues.

Some clinicians within the institution, but outside the group that authored the guideline, may well disagree with the plan. This is particularly relevant in the setting of trisomy 13 and 18, where standards are evolving, and opinions are often strongly held. Given the disparity of opinions on this matter, it seems right to give colleagues outside the division significant latitude, as specified below.

\section{Risks of the process}

This process of guideline development in difficult clinical scenarios has many positive attributes, but there are risks as well. Groupthink - a phenomenon whereby dysfunctional decision-making occurs in a group that prizes conformity over critical evaluation of individual viewpoints - is a significant concern, especially if there is one or a few dominant voices in the discussion. When important decisions are made in a group setting, groupthink can lead to a consensus on a plan that the individual members might not, on their own, have considered wise [33]. Individuals might be influenced by the perceived (perhaps falsely) opinion of peers held in high regard. One safeguard against this concern could include encouraging people to raise and seriously consider any potential counterarguments to the plan, even if all seem at the outset to agree. Also, two meetings separated by some time, even if only one or two nights, might allow for solitary contemplation. One might find that, what sounded appropriate when a room full of colleagues agreed, does not seem as good when considered in isolation. That should serve as a red flag to the individual and should subsequently be shared with the group.

Another risk of the process is the cognitive dissonance associated with a major change in practice, making some psychologically more likely to favor the status quo. A preference for a plan that differs from longstanding practice may carry with it the implication (or cognition) for practitioners that this then means they have been doing something unethical up to now. This could make it difficult to change established practice, even if the ethical arguments seem to favor the change, and can be an especially difficult challenge to overcome when associated with life-or-death decisions. It may be psychologically easier for one to confirm the validity of past practice, rather than be left with the perception that one has been "doing it wrong." The best safeguard against this is awareness that moral progress in medicine, as elsewhere, will require us to revisit and sometimes alter our judgements and our practice. Good, experienced physicians rightfully do many things differently than they did years ago. This does not suggest a lack of professional competence in the past, but rather it suggests progress in the profession, and a recognition that we should always be open to doing things better. A willingness to change should not be viewed as an accusation regarding past practice, but rather as a healthy attitude toward progress.

\section{Suggested policy}

Different groups of thoughtful individuals may go through the process described above and come to different conclusions. We feel, however, that going through this or a similar process is advisable. It is not here suggested that the policy ultimately adopted at the authors' institution is the only correct one, or that it should be adopted by others. Rather, in the spirit of transparency, our approach is shared below.

Prior to the current iteration of a process such as this at Yale New Haven Children's Hospital, a majority of neonatologists had agreed to offer comfort care only to patients with these aneuploidies, which did not include medically administered nutrition and hydration, medications for management of cardiac lesions, or respiratory support of any kind. After the process of guideline development described above, there was a change in approach. The Division of Neonatology decided that medically indicated interventions will be made available to newborns with trisomy 13/18, after ensuring that parents are well informed of risks and benefits. This includes interventions previously withheld such as positive pressure ventilation, medically administered nutrition and hydration, and surgical repair of problems such as congenital heart disease. Transplant candidacy was not addressed at this time so would require significant discussion, and likely consultation with the hospital ethics committee, if a case were to arise within the institution, to determine the appropriateness of such a complicated intervention.

An important aspect of this approach is that it reflects what the neonatologists felt should be offered. It is not intended to obligate others, such as surgeons, to provide procedures they do not feel are appropriate. If neonatologists believe that a given patient could benefit from a surgical procedure, this is discussed with the relevant surgeons in the institution. If a surgeon agrees, the procedure is then offered to parents. If the parents request a medically indicated procedure but the relevant surgeons in the institution choose not to do it on ethical grounds or concerns regarding medical complexity, the patient is supported in the preoperative period while the medical team makes a good faith effort to locate a surgeon and facility elsewhere willing to 
provide the procedure. If the diagnosis is made prenatally, these discussions could result in a decision to deliver the patient in a different facility willing to provide the requested interventions.

There was a clear distinction made by the group between what should be offered to families of children with trisomy $13 / 18$, and what would be recommended. As with many other patients with similarly poor prognoses, most of the physicians who were involved in this discussion generally recommend to parents a focus on comfort care only but are willing to offer other interventions described above to wellinformed parents, and defer to their right to parental authority.

\section{Conclusion}

A consideration of available outcome data, and the arguments for and against the ethical permissibility of medical and surgical treatments beyond comfort measures in the setting of a fetus or newborn with trisomy 13 or 18 , suggests that some treatments formerly not made available for these patients should be seen as ethically permissible. Therefore, parents of these patients should be given more options than has traditionally been the case in many medical centers. Our approach to these patients, as with all newborn patients, should be directed by our best understanding of prognosis, feasibility, ethical considerations, and the wishes and values of well-informed parents. Every institution that manages such newborns should develop a written guideline based on these same considerations, in an effort to provide a fair and transparent approach. And, as with any other clinical guideline, it should be revisited periodically as outcomes data and professional values continue to evolve.

\section{Compliance with ethical standards}

Conflict of interest The authors declare that they have no conflict of interest.

\section{References}

1. Springett A, Wellesley D, Greenlees R, Loane M, Addor M-C, Arriola L, et al. Congenital anomalies associated with trisomy 18 or trisomy 13: a registry-based study in 16 European countries, 2000-2011. Am J Med Genet Part A. 2015;167A:3062-9.

2. Edwards JH, Harnden DG, Cameron AH, Crosse VM, Wolff OH. A new trisomic syndrome. Lancet. 1960;1:787-9.

3. Patau K, Smith D, Therman E, Inhorn S, Hans P. Multiple congenital anomaly caused by an extra autosome. Lancet. 1960;275:790-93.

4. Koogler TK, Wilfond BS, Ross LF. Lethal language, lethal decisions. Hastings Cent Rep. 2003;33:37-41.

5. Meyer RE, Liu G, Gilboa SM, Ethen MK, Aylsworth AS, Powell CM, et al., for the National Birth Defects Prevention Network.
Survival of children with trisomy 13 and trisomy 18: a multi-state population-based study. Am J Med Genet Part A. 2015;9999A: $1-13$.

6. Maeda J, Yamagishi H, Furutani Y, Kamisago M, Waragai T, Oana $\mathrm{S}$, et al. The impact of cardiac surgery in patients with trisomy 18 and trisomy 13 in Japan. Am J Med Genet Part A. 2011;155:2641-46.

7. Mercurio M. Physicians' refusal to resuscitate at borderline gestational age. J Perinatol. 2005;25:685-9.

8. Nelson KE, Rosella LC, Mahant S, Guttmann A. Survival and surgical interventions for children with trisomy 13 and 18. JAMA. 2016;316:420-8.

9. Janvier A, Farlow B, Wilfond BS. The experience of families with children with trisomy 13 and 18 in social networks. Pediatrics. 2012;130:293-8.

10. Bruns DA. Developmental status of 22 children with trisomy 18 and eight children with trisomy 13: implications and recommendations. Am J Med Genet A. 2015;167A:1807-15.

11. Guon J, Wilfond BS, Farlow B, Brazg T, Janvier A. Our children are not a diagnosis: the experience of parents who continue their pregnancy after a prenatal diagnosis of trisomy 13 or 18 . Am J Med Genet Part A. 2014;164A:308-18.

12. Nelson KE, Hexem KR, Feudtner C. Inpatient hospital care of children with trisomy 13 and trisomy 18 in the United States. Pediatrics. 2012;129:869-76.

13. Peterson JK, Kochilas LK, Catton KG, Moller JH, Setty SP. Long-term outcomes of children with trisomy 13 and 18 after congenital heart disease interventions. Ann Thorac Surg. 2017;103:1941-9.

14. Costello JP, Weiderhold A, Louis C, Shaughnessy C, Peer SM, Zurakowski D, et al. A contemporary, single-institutional experience of surgical versus expectant management of congenital heart disease in trisomy 13 and 18 patients. Pediatr Cardiol. 2015;36:987-92.

15. Kaneko Y, Kobayashi J, Yamamoto Y, Yoda H, Kanetaka Y, Nakajima $Y$, et al. Intensive cardiac management in patients with trisomy 13 or trisomy 18 . Am J Med Genet Part A. 2008;146A:1372-80.

16. Niermeyer S, Kattwinkel J, Van Reempts P, Nadkarni V, Phillips $\mathrm{B}$, Zideman $\mathrm{D}$, et al. International Guidelines for Neonatal Resuscitation: An Excerpt From the Guidelines 2000 for Cardiopulmonary Resuscitation and Emergency Cardiovascular Care: International Consensus on Science Contributors and Reviewers for the Neonatal Resuscitation Guidelines. Pediatrics. 2000;106: e29.

17. International Liaison Committee on Resuscitation. Part 7: Neonatal resuscitation. Resuscitation. 2005;67:293-303.

18. Kattwinkel J, Perlman JM, Aziz K, Colby C, Fairchild K, Gallagher J, et al. Part 15: neonatal resuscitation: 2010 American Heart Association Guidelines for Cardiopulmonary Resuscitation and Emergency Cardiovascular Care. Circulation. 2010;122:S909-S919.

19. Wyckoff MH, Aziz K, Escobedo MB, Kapadia VS, Kattwinkel J, Perlman JM, et al. Part 13: neonatal resuscitation: 2015 American Heart Association Guidelines Update for Cardiopulmonary Resuscitation and Emergency Cardiovascular Care. Circulation. 2015;132 Suppl 2:S543-S560.

20. Bosslet GT, Pope T, Rubenfeld GD, et al. An Official ATS/ An Official ATS/AACN/ACCP/ESICM/SCCM Policy Statement: responding to requests for potentially inappropriate treatments in intensive care units. Am J Respir Crit Care Med. 2015;191:1318-30.

21. Mercurio MR. The aftermath of baby doe and the evolution of newborn intensive care. Ga State Univ Law Rev. 2012;25:835-63.

22. Chapman RL, Peterec SM, Bizzarro MJ, Mercurio MR. Patient selection for neonatal extracorporeal membrane oxygenation: beyond severity of illness. J Perinatol. 2009;29:606-11. 
23. Adams RC, Levy SE. Council on children with disabilities. Shared decision-making and children with disabilities: pathways to consensus. Pediatrics. 2017;139:e20170956.

24. Jardine J, Glinianaia SV, McConachie H, Embleton ND, Rankin J. Self-reported quality of life of young children with conditions from early infancy: a systematic review. Pediatrics. 2014;134: e1129.

25. Kosiv KA, Gossett JM, Bai S, Collins RT. Congenital heart surgery on in-hospital mortality in trisomy 13 and 18 . Pediatrics. 2017;140:e20170772.

26. Beauchamp TL, Childress JF. Principles of biomedical ethics. 7th ed. New York: Oxford University Press; 2013.

27. Rawls J. 1971, A theory of justice. 2nd ed. Cambridge: Harvard University Press; 1999.

28. AAP Committee on Bioethics. Informed consent in decisionmaking in pediatric practice. Pediatrics. 2016;138:e20161484.
29. Weise KL, Okun AL, Carter BS, et al. Guidance on forgoing lifesustaining medical treatment. Pediatrics. 2017;140:e20171905.

30. Hayman WR, Leuthner SR, Laventhal NT, Brousseau D, Lagatta JM. Cost comparison of mechanically ventilated patients across the age span. J Perinatol. 2015;35:1020-6.

31. Rosenfeld RM, Shiffman RN. Clinical practice guideline development manual: a quality-driven approach for translating evidence into action. Otolaryngol Head neck Surg. 2009;140 Suppl 1:S1-43.

32. Lewis-Newby M, Wicclair M, Pope T, Rushton C, Curlin F, Diekema D, et al., ATS Ethics and Conflict of Interest Committee An official American Thoracic Society policy statement: managing conscientious objections in intensive care medicine. Am J Respir Crit Care Med. 2015;191:219-27.

33. Janis IL. Victims of groupthink: a psychological study of foreign policy decisions and fiascoes. Boston, MA: Houghton Mifflin Company; 1972. 